\section{SEPTEMBER 2006}

$10^{\text {th }}$ National Forest Congress: Sustainable Forest Management in the Boreal - A Global Challenge. 24-27 September, Gatineau-Ottawa. 1-866-441-4006 www.nfc-cfn.ca

Poplar Council of Canada Joint Meeting with Poplar Council of the United States, the US Short-Rotation Woody Crops Working Group and IUFRO Working Group on ShortRotation Crops 25-28 September Pasco, Washington State, USA. Call for papers at: www.poplar.ca/pdf/pasco2006 Info: www.poplar.ca

\section{OCTOBER 2006}

The Lake Abitibi Model Forest and the Canadian Institute of Forestry are proud to host:

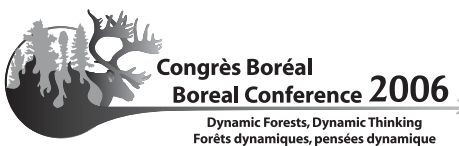

The Boreal Conference 2006

1-4 October

with optional field trips Oct. 5 \& 6

Cochrane / Moosonee /

Moose Factory, Ontario.

www.borealconference2006.ca
Leading Edge 2006 - Niagara Escarpment Commission's Conference on Sustainability, Healthy Communities and Biosphere Research.

04-06 October, Burlington, Ontario. www.escarpment.org

Sustainable Forest Management with Fast Growing Plantations.

10-13 October,

Charleston, North Carolina. www.iufro.org/science/divisions/ division-4

Seventh Canadian Urban Forestry Conference - “The Tree at the Centre of Urban Development" 11-13 October, Quebec City. Contact: Michael Rosen at mrosen@treecanada.ca

\section{Colloque Eastern CANUSA} sur les sciences forestières/Eastern CANUSA Forest Science Conference 19-21 octobre/October Pavillon Alphonse Desjardins Université Laval Québec (Québec) www.mrnf.gouv.qc.ca/ecanusa

\section{NOVEMBER 2006}

Today's Silviculture: Tomorrow's Forest. Symposium of the Forestry \& Industrial Vegetation Management Working Group of the Canadian Weed Science Society.

28-29 November, Victoria, B.C.

www.forestry.cwss-scm.ca/index.html

\section{MARCH 2007}

2nd Fire Behaviour and Fuels Conference - Fire Behaviour Fundamentals and Applications 26-30 March, San Diego. www.iawfonline.org

\section{JULY 2007}

Complex Stand Structures and Associated Dynamics: Measurement Indices and Modeling Approaches. (IUFRO 4.01.02) 29 July - 02 August, Sault Ste. Marie, Ontario Contact: pnewton@nrcan.gc.ca www.iufrosault.org 